\title{
Defining the function of a novel TNF receptor superfamily member
}

\author{
Joseph Atumonye ${ }^{1}$, Matthew J. Turner ${ }^{2,3}$, Hongming Zhou ${ }^{2}$, Radomir Slominski ${ }^{2}$, Avery \\ Dawes $^{4}$ \\ ${ }^{1}$ Indiana University School of Medicine, ${ }^{2}$ Indiana University School of Medicine, Department of \\ Dermatology, Richard L. ${ }^{3}$ Roudebush VA Medical Center, ${ }^{4}$ Indianapolis, IN, Indiana University \\ School of Medicine Wells Center Internship.
}

\section{Background:}

Insulin Growth Factor Like 1 Receptor (IGFLR1) is a putative TNF receptor superfamily member of unknown function. Transcripts for IGFLR1 are most abundant in T cells in mice and several leukocyte subsets in humans. A major limitation to understanding IGFLR1 function is the lack of biochemical data regarding IGFLR1 expression and processing in leukocytes.

\section{Experimental Design:}

A panel of human leukocyte and keratinocyte cell lines was screened for IGFLR1 transcript expression by quantitative PCR. Immunoblotting was then used to detect IGFLR1 protein and characterize which isoform(s) were expressed. Chimeric IGFLR1 construct that could be used to mimic IGFLR1 activation was also generated. This chimeric protein, hNGFR-DmrB2- hIGFLR, contains two DmrB domains that allow for multimerization in the presence of AP20187.

\section{Results:}

IGFLR1 transcript expression varied between hematopoietic cell lines with relatively low expression in MOLT4 cells ( $T$ cell line), intermediate expression in IM9 and ARH77 cells (B cell lines) and high expression in MKB cells ( $T$ cell line). Immunoblotting in these cell lines revealed a similar protein signal expression pattern. Immunoblotting also suggested that the most abundant isoform of IGFLR1 was likely isoform c, which lacks ligand-binding and transmembrane domains. With respect to the hNGFR-DmrB2-hIGFLR1 construct, the chimeric protein was detected in $\mathrm{CHO}$ cells by immunoblotting and biochemical analyses confirmed that addition of AP20187 induced multimerization of this protein. These findings confirm the functionality of hNGFR-DmrB2-hIGFLR1.

\section{Potential Impact:}

Biochemical studies of endogenously expressed IGFLR1 in these cell lines suggest differential mRNA splicing may play an important role in the production and function of IGFLR1 isoforms. Future studies using the hNGFR-DmrB2-hIGFLR1 construct in the aforementioned human cell lines can be performed to study IGFLR1 signaling. 